\title{
The Biological Assay of Streptomycin by a Modified Cylinder Plate Method
}

\author{
By K. A. BROWNLEE, C. S. DELVES, M. DORMAN, C. A. GREEN, \\ E. GRENFELL, J. D. A. JOHNSON AND N. SMITH \\ The Research Department of The Distillers Company Limited, Great Burgh, \\ Epsom, Surrey
}

SUMMARY: The use of plate glass sheets carrying twenty-four cylinders in place of Petri dishes for the biological assay of streptomycin by the cylinder-plate method initially led to large assay errors. The substitution of cavities for cylinders, of a single layer of agar for two layers, one sterile and one inoculated, of the four-point assay design for 'reading off the curve', and of an optical projection method of measurement diminished the fiducial limits of error of an assay to $80-125 \%(P=0.95)$.

Over the range of concentrations $0 \cdot 1-100 \cdot 0$ units streptomycin $/ \mathrm{ml}$. the deviations from linearity of the graph of zone diameter against the logarithm of the concentration were very small.

Variations in thickness of the agar layer and in the intensity of inoculation had a marked effect on zone diameter.

An $8 \times 8$ Latin square lay-out reduced the internal error of an assay to $91-110 \%$ $(P=0.95)$, and with this increased accuracy a bias in the results was revealed arising from the time interval between filling successive cavities on the plate. This time effect was eliminated by the adoption of an appropriate order of filling, with a quasi-Latin square lay-out. The form of the assay finally adopted had fiducial limits of error, estimated both internally and externally, of about $95-105 \%(P=0.95)$.

During the 1939-45 War it became increasingly difficult to procure adequate supplies of Petri dishes for the assay of penicillin on the well-known cylinder plate, and attempts were therefore made to use plate-glass sheets carried on wooden or aluminium frames as substitutes for the dishes. These attempts proved quite successful for plant control.

Wider variations than those permissible for research purposes, obtained when these frames were used for the biological assay of streptomycin, necessitated a closer examination of the factors which might influence the accuracy of the result. This paper is concerned solely with certain features which were found to influence the assay as carried out when using frames.

\section{Initial techniques}

The frames were either of hardwood or aluminium, the latter being the more desirable because they do not warp. Even so, it is somewhat difficult to get a frame which does not leak slightly, and because of possible variations in the amount of agar left on a plate with consequent variation in the size of the zones of inhibition, each plate was made a self-contained assay, thus avoiding reference of an unknown to a standard on another plate.

In early work the assays were made on plate-glass sheets, $32 \times 14 \mathrm{~cm}$., held in a frame whose inside measurements were $27 \frac{1}{2} \times 10 \mathrm{~cm}$. The nutrient agar was approximately $2 \mathrm{~mm}$. deep and supported twenty-four porcelain cylinders 
of the well-known type. The twenty-four cylinders were arranged in three staggered rows of eight according to Table 1 , where $S_{H}$ represents the cylinders carrying the standard of higher concentration ( 40 units $/ \mathrm{ml}$.), $S_{L}$ those carrying the standard of lower concentration (4 units/ml.), and $U_{1}$ and $U_{2}$ represent cylinders carrying solutions of unknown concentration. The plates were sterilized by dry heat at $160^{\circ}$, allowed to cool to about $80-90^{\circ}$ and filled first with $60 \mathrm{ml}$. of sterile nutrient agar, and as soon as it was set, by $60 \mathrm{ml}$. of nutrient agar at $60^{\circ}$ previously inoculated with Bacillus subtilis. As each layer of agar was poured the plate was placed on a level bench covered with plate glass, so that a double layer of agar of uniform thickness resulted.

\section{Table 1. Plate lay-out for two unknown solutions using} rectangular plates and 'reading off the curve'

$$
\begin{array}{cccccccccc}
S_{L} & U_{1} & U_{2} & S_{H} & U_{2} & S_{L} & S_{H} & U_{1} \\
S_{H} & S_{L} & U_{1} & U_{2} & U_{1} & U_{2} & S_{L} & S_{H} \\
U_{2} & S_{H} & S_{L} & U_{1} & S_{H} & U_{1} & U_{2} & S_{L}
\end{array}
$$

The porcelain cylinders were sterilized by heating on a hot plate, picked up with sterile tweezers and dropped from a height of a few mm. on to the agar in the desired positions. The cylinders were then filled with the various solutions; the following precautions being taken:

(a) One person filled the cylinders on a given plate.

(b) The same filler (of the fountain-pen filler type) was used for all solutions.

(c) The same number of drops was used in each case.

(d) As far as possible the tip of the filler was held at the same height above the cylinders, to prevent the drop from touching the cylinders before becoming detached and to avoid the formation of 'air-bells', and the filler was held at the same angle (nearly vertical).

The potencies of the unknown solutions were estimated in the usual manner by 'reading off the curve'. Such precautions, amongst others, are commonly believed to lead to good assay results, but a check on the method, all operators being aware that it was being made, showed that twenty assays on the same sample could give results as widely discrepant as $5 \cdot 8$ and $19 \cdot 5$ units $/ \mathrm{ml}$.

\section{Modifications in technique}

(a) The use of cavities in place of cylinders. A number of features of the use of cylinders was considered undesirable, in particular, their variation in size and the variation in the depth to which they penetrated the agar. Cylinders which penetrated deeply into the agar would reduce the area of the zone through which the streptomycin solution would initially diffuse. Accordingly cavities were tried.

The plate was poured and allowed to set. After about $30 \mathrm{~min}$. at room temperature the cavities were bored in the agar with a sterile no. 5 cork borer which was clean, sharp and free from burred edges. The agar disks were then removed with small sterilized spear-shaped needles. These were better than 
plain needles because the disks, once impaled, do not slip off, and a number can be impaled one after the other.

At first the test solutions crept between the agar and the glass plate, but this trouble was not encountered later.

For ready calculation of the internal errors of the assays the standard fourpoint assay method was used (e.g. Finney, 1944). In this the standard is used at two dilutions, and the unknown is also used at two dilutions, the dilution ratio being the same for both standard and unknown. The same lay-out as in Table 1 was used, with the modification that each plate carried only one unknown, and $U_{1}$ and $U_{2}$ represented its upper and lower dilutions.

A rough experiment showed that the accuracy with cavities was certainly no worse than that obtained with cylinders and was possibly better, so cavities were adopted forthwith.

(b) The use of a single layer of agar. It was found more convenient to pour the agar already inoculated on to the plate in one layer. This tended to produce a double edge to the zones of inhibition (not to be confused with a 'halo') for reasons which are not clear. The larger zone is on the upper surface of the agar, the smaller zone at the glass-agar interface. Experiment showed, however, that provided one or the other was chosen for measurement and the choice maintained there was no difference in the potencies obtained or in the standard error of their determinations.

(c) Method of measurement of diameters of zones of inhibition. At first, measurements of zone diameter were made with calipers, transparent rulers or a travelling microscope. The first two methods are not accurate enough and were, by comparison with the method now used, time-consuming and laborious. The travelling microscope was accurate but even more irksome to use. In our present apparatus, the zones are magnified approximately seven times without loss of definition, and the readings can be made almost as fast as they can be written down. Illumination is from a photoflood lamp in the top portion of the apparatus, and the light passes downwards through a condenser lens about 6 in. in diameter and of 3 in. focal length. The plates are placed beneath the lens and the transmitted beam then passes on to a $9 \mathrm{in}$. focal length anastigmatic portrait lens arranged below. At the bottom of the apparatus is a $6 \times 6$ in. mirror slightly tilted so as to throw the reflected image slightly forward and upwards on to the ground-glass screen. The apparatus may be extemporized from an ordinary projection lantern arranged vertically, the plates being inserted in the position normally occupied by the lantern slides. In all the experiments described below zone-diameter measurements are those obtained on such apparatus. Typical magnified zones have diameters of $10-20 \mathrm{~cm}$., and the edges are quite sharp. These modifications reduced the $95 \%$ fiducial limits of error of an assay to about $80-125 \%$.

\section{The bases of the assay}

(a) The linearity of relationship between zone diameter and logarithm of concentration. The linearity of the relationship between zone diameter and the logarithm of the concentration was tested on twenty plates each containing 
$60 \mathrm{ml}$. of agar and carrying twenty-four cavities. On each plate four cavities were filled with each of six solutions containing $0 \cdot 1,0 \cdot 25,0 \cdot 5,1 \cdot 0,10 \cdot 0$ and 100.0 units of streptomycin sulphate $/ \mathrm{ml}$. in $\mathrm{pH} 8.0$ buffer. The average zone diameters are given in Table 2 . The best straight line and the best parabola were fitted to the points at $0 \cdot 1,1 \cdot 0,10 \cdot 0$ and $100 \cdot 0$. The linear coefficient for the parabola was $3 \cdot 659 \pm 0 \cdot 044$, and the quadratic coefficient $0 \cdot 0796 \pm 0 \cdot 0492$. The quadratic coefficient thus cannot be considered significant $(P=0 \cdot 1)$. It is clear that the curvature over this wide range of concentration, even if it does exist, is nevertheless so small as to be of no consequence for practical purposes. The values for the two end-points $(0 \cdot 1$ and $100 \cdot 0)$ given by the best straight line and the best parabola have been inserted in Table 2 to show the smallness of the difference between the fit of the two curves.

Table 2. Relation between zone diameter $\left(\mathrm{cm} .^{*}\right)$ and logarithm of concentration

\begin{tabular}{|c|c|c|c|c|c|c|c|c|}
\hline & & & \multicolumn{6}{|c|}{ Concentration (units/ml.) } \\
\hline & & & $0 \cdot 1$ & $0 \cdot \dot{25}$ & 0.5 & $1 \cdot 0$ & $10 \cdot 0$ & $100 \cdot 0$ \\
\hline $\begin{array}{l}\text { Observed average } \\
\text { meter* }\end{array}$ & zone & dia- & $11 \cdot 38$ & $12 \cdot 78$ & $13 \cdot 99$ & $15 \cdot 30$ & $18 \cdot 81$ & $22 \cdot 41$ \\
\hline $\begin{array}{l}\text { Value calculated } \\
\text { straight line }\end{array}$ & from & best & $11 \cdot 484$ & - & 一 & - & 一 & $22 \cdot 461$ \\
\hline $\begin{array}{l}\text { Value calculated } \\
\text { parabola }\end{array}$ & from & best & $11 \cdot 405$ & - & - & - & - & $22 \cdot 382$ \\
\hline
\end{tabular}

* The measurements refer to the zone diameters on the screen of the measuring apparatus, which magnified about seven times.

(b) Effect of uniformity of the agar layer. The $95 \%$ fiducial limits for the assay as carried out with the modification outlined above were approximately 80-125\%. This, though a substantial improvement on the earlier figures, was not good enough.

A factorial experiment was made to determine the effects of variation in the thickness of the agar and density of inoculation. The quantity of agar used was at two levels, normal and three times the normal, and the intensity of inoculation was at two levels, normal and four times the normal. Two plates were used at each of the four combinations of quantity of agar and intensity of inoculation, and three concentrations of streptomycin, 1,5 and 10 units $/ \mathrm{ml}$., tested on each plate. The average diameters of the zones of inhibition are given in Table 3.

Table 3. Average diameter of zones (cm.) of inhibition for different intensities of inoculation and quantities of agar

Intensity of inoculation

$\overbrace{1}^{\text {Streptomycin units } / \mathrm{ml} .} \begin{gathered}\text { Normal } \\ \text { S }\end{gathered} \overbrace{10 \text { Average }}^{\text {Avera }}$

Quantity of agar:

$\begin{array}{llllllllll}\text { Normal } & 15 \cdot 48 & 16 \cdot 92 & 17 \cdot 85 & 16 \cdot 75 & 14 \cdot 10 & 15 \cdot 29 & 16 \cdot 16 & 15 \cdot 18 & 15 \cdot 96 \\ \text { Treble } & 14 \cdot 01 & 15 \cdot 29 & 16 \cdot 18 & 15 \cdot 16 & 12 \cdot 01 & 12 \cdot 96 & 13 \cdot 96 & 12 \cdot 98 & 14 \cdot 07 \\ \text { Average } & & & & 15 \cdot 95 & & & & 14 \cdot 08 & \end{array}$


To test the significance of these results we need the appropriate analysis of variance. This is rather unusual, as the error term for testing the effects involving the concentration is different from and much smaller in magnitude than that for testing the main effects of intensity of inoculation and quantity of agar and their mutual interaction (Table 4).

Table 4. Analysis of variance of experiment on variation in quantity of agar and intensity of inoculation

Source of variance

Intensity of inoculation $(I)$

Quantity of agar (Q)

Interaction of $I$ and $Q$

Error term for $I, Q$ and $I Q$

Concentration of solution $(C)$

Interaction of $C$ with $I$

Interaction of $C$ with $Q$

Interaction of $C$ with $I Q$

Error term for above four terms involving $C$

Total

\begin{tabular}{|c|c|c|c|}
\hline $\begin{array}{l}\text { Degrees of } \\
\text { freedom }\end{array}$ & $\begin{array}{l}\text { Sums of } \\
\text { squares }\end{array}$ & $\begin{array}{c}\text { Mean } \\
\text { squares }\end{array}$ & $\boldsymbol{P}$ \\
\hline 1 & $2147 \cdot 042$ & $2147 \cdot 04,2$ & 0.05 \\
\hline 1 & $2147 \cdot 042$ & $2147 \cdot 042$ & 0.05 \\
\hline 1 & $57 \cdot 042$ & $57 \cdot 042$ & $>0.05$ \\
\hline 4 & $101 \cdot 832$ & $25 \cdot 458$ & - \\
\hline 2 & $1838 \cdot 384$ & $919 \cdot 292$ & $<0.001$ \\
\hline 2 & 8.583 & $4 \cdot 292$ & $>0.05$ \\
\hline 2 & $4 \cdot 083$ & $2 \cdot 042$ & $>0.05$ \\
\hline 2 & $1 \cdot 083$ & 0.542 & $>0.05$ \\
\hline 6 & $7 \cdot 918$ & $1 \cdot 320$ & - \\
\hline 21 & $6314 \cdot 950$ & & \\
\hline
\end{tabular}

The concentration of solution effect $(C)$ is of course significant, but it is interesting to note that its interactions with $I$ (intensity of inoculation) and $Q$ (quantity of agar) are completely non-significant. That is to say, the slope (the increase in zone diameter with the logarithm of the concentration) is not significantly different for the two levels of intensity of inoculation and for the two levels of quantity of agar.

Both the intensity of inoculation and quantity of agar effects are highly significant, but their interaction is not significant. We can therefore conclude that they operate independently. Their magnitudes for the changes concerned, namely, four-fold for intensity of inoculation and three-fold for quantity of agar, are approximately the same, nearly $2 \mathrm{~cm}$. on an average of $15 \mathrm{~cm}$.

\section{The Latin square and quasi-Latin square lay-out}

The Latin square principle. From the experiment described in the previous section it was apparent that one possible source of error lay in the variations in the thickness of the agar; variations in the intensity of inoculation should of course not occur when working with a single layer of agar. Although the lay-out given in Table 1 was an attempt to give a balanced arrangement, plates of this shape will not accommodate a lay-out which would allow the effects of inhomogeneity in the agar to be removed from the error. Plates $35 \times 35 \mathrm{~cm}$. with aluminium frames $27 \frac{1}{2} \times 27 \frac{1}{2} \mathrm{~cm}$. (inside measurement) were constructed to accommodate sixty-four cavities laid out in the form of an $8 \times 8$ square. Each row contained solutions $S_{H}, S_{L}, 1_{H}, 1_{L}, 2_{H}, 2_{L}, 3_{H}, 3_{L}$, where $S$ is the standard and 1, 2 and 3 are three unknown solutions, and the suffixes $H$ and $L$ denote the higher and lower concentration, a ratio of 10 to 1 being employed throughout. The use of the Latin square arrangement (Fisher, 
1942) made it possible to have all eight treatments occurring once and only once in each row and also once and only once in each column. Differences between columns were thus independent of differences between rows and between treatments, differences between rows were independent of differences between columns and treatments, and, most important, differences between treatments were independent of differences between rows and between columns. Thus effects due to one or more rows and columns having agar different in thickness from the others did not introduce any error into the determination of the average zone diameters for $S_{H}, S_{L}, 1_{H}$, etc.

The use of this Latin square had the following general advantages over the earlier methods :

(a) An assay of three unknowns required sixty-four cavities instead of seventy-two with the earlier rectangular plates. Even with this decrease in total number of cavities, the number of observations at each point actually increased to eight instead of six as on the smaller plates.

(b) It was as easy and the plates took no longer to pour than smaller ones. Washing also took very little longer.

(c) The internal error of an assay was much reduced, the $95 \%$ fiducial limits being about 91 and $110 \%$. These limits being rather less than half those of the earlier form, one of these Latin square assays was therefore equivalent to over four of the earlier ones.

Effect of time in filling the plates. Working with assays of this type, the much increased accuracy revealed a bias in the potencies obtained, in that the 'unknowns', when they were actually known, came out lower than expected.

It is well known that if inoculated plates are kept in the refrigerator before use, the zones of inhibition are much larger than ordinarily, probably because the growth of the bacteria is checked, or some of the bacteria are killed, even though the plates are given ample time to warm up to room temperature before filling. The influence of the time the plates stood before filling was accordingly tested (time of standing before filling). Another possible source of error was the variation in the length of time the inoculated agar was held at a high temperature before pouring into the plates (age of agar). Eighteen plates were, therefore, poured in turn and numbered as they were poured. Plates 1, 2 and 3 were regarded as being $2 \mathrm{~min}$. old; 4, 5 and 6 as $4 \mathrm{~min}$.; and so on. The time of standing was also regarded from a similar standpoint. The total time for pouring was of the order of $10 \mathrm{~min}$., which might have had an appreciable effect on an inoculated agar held at $60^{\circ}$ for 10-20 min. beforehand, but which would be negligible, we presumed, compared with the 1-3 hr. which would elapse before the plates were filled. The key to the experiment, together with the results, is given in Table 5 .

All the test solutions contained $10 \mathrm{units} / \mathrm{ml}$. However, one was regarded as the standard and went on the plate first in the first series of nine plates and last in the second series of nine plates. The other three were regarded as separate samples and placed on the plates in the order shown by the prefixes A, B and C.

In the first nine experiments the concentrations of the second, third and fourth solutions are expressed in terms of the first as standard; in the last 
nine experiments the first, second and third are expressed in terms of the last as standard. (Two plates, 5 and 17 , were upset and unfortunately could not be included.)

The results are very striking. Collectively, they indicate that the time which elapses between filling with one sample and the next is of vital importance to the assay result. In almost every case there is a uniform drop from sample to sample. (It should be noted that plate 18 was the fourth plate filled by operator C, i.e. it marked the point where she changed from 'standard first'

Table 5. Effect of time of filling, and other factors, on potency estimations

$\begin{array}{ccc}\text { Plate } & \text { Operator } & \begin{array}{c}\text { Approx. } \\ \text { age of agar } \\ \text { (min.) }\end{array} \\ 1 & A & 2 \\ 2 & B & 2 \\ 3 & C & 2 \\ 4 & A & 4 \\ 5 & B & 4 \\ 6 & C & 4 \\ 7 & A & 6 \\ 8 & B & 6 \\ 9 & C & 6 \\ 10 & A & 8 \\ 11 & B & 8 \\ 12 & C & 8 \\ 13 & A & 10 \\ 14 & B & 10 \\ 15 & C & 10 \\ 16 & A & 12 \\ 17 & B & 12 \\ 18 & C & 12\end{array}$

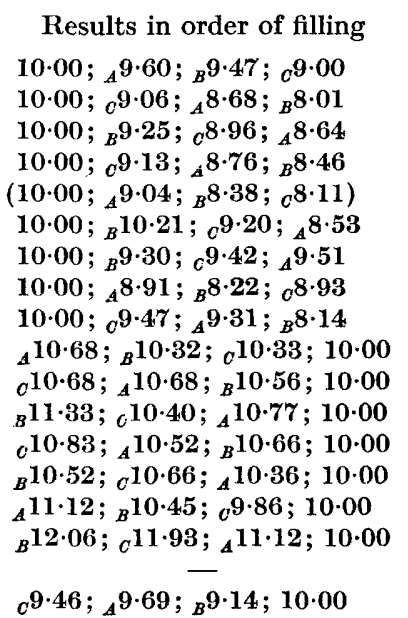

Approx. time of standing before filling (min.)

The figures for plate 5 are the values inserted for the missing values. The prefixes $A, B$ and $C$ relate to the three samples.

to 'standard last' and having regard to the fact that the whole experiment had had to be repeated because of a misunderstanding, we are inclined to think that it was filled erroneously.) Table 6 gives an analysis of variance for the first nine plates. The values for the missing plate were inserted by a method based on

Table 6. Analysis of variance of logarithms of results in Table 5

\begin{tabular}{|c|c|c|c|c|c|}
\hline Source of variance & $\begin{array}{l}\text { Degrees } \\
\text { of } \\
\text { freedom }\end{array}$ & $\begin{array}{c}\text { Sums of } \\
\text { squares } \\
\left(\times 10^{6}\right)\end{array}$ & $\begin{array}{c}\text { Mean } \\
\text { squares } \\
\left(\times 10^{6}\right)\end{array}$ & $\boldsymbol{F}$ & $\boldsymbol{P}$ \\
\hline Operators & 2 & $4334 \cdot 740$ & $2167 \cdot 370$ & 4.56 & $0 \cdot 05$ \\
\hline Time of standing before filling & 2 & $815 \cdot 629$ & $407 \cdot 814$ & - & - \\
\hline Age of agar & 2 & $274 \cdot 073$ & $137 \cdot 036$ & 一 & - \\
\hline \multicolumn{6}{|l|}{ Order of filling: } \\
\hline Linear term & $\mathbf{1}$ & $4608 \cdot 000$ & $4608 \cdot 000$ & $9 \cdot 69$ & $0 \cdot 01$ \\
\hline Quadratic term & $\mathbf{I}$ & $249 \cdot 185$ & $249 \cdot 185$ & - & - \\
\hline Samples & $\mathbf{2}$ & $407 \cdot 629$ & $203 \cdot 814$ & - & - \\
\hline Residual & 13 & $6181 \cdot 262$ & $475 \cdot 482$ & - & - \\
\hline Total & 23 & $16870 \cdot 518$ & & & \\
\hline
\end{tabular}


the principle of least squares. Having inserted these, the total degrees of freedom become three less than otherwise, and this decrease of three occurs in the residual.

The results have been analysed on the assumption that the second, third and fourth results on each of the first nine plates have been assayed in terms of the first sample placed on each plate.

The sample effect is non-existent, as also are the order of taking the plate and the age of agar at pouring. The order of filling has its linear component (expressed in terms of logarithms to base 10) highly significant, but its curvature non-significant. The operator effect is significant at the $5 \%$ level. One particular operator had a particularly slow-acting filler and took much longer as a consequence in filling her plates. This serves to emphasize the effect of the time which elapses during filling on the assay result.

The residual variance averaged over this experiment and a similar one is approximately 480 , corresponding to a standard deviation of about $\mathbf{2 2}$. The units in which the analysis has been conducted have been logarithms to base $10 \times 1000$ (this transformation being made for simplicity in the computation). Accordingly, a standard deviation in the real units is 0.022 , and when converted to the $95 \%$ fiducial limit on an expected figure of 100 the upper limit is about 111. This is very close to the estimate made for the Latin square when first used.

It was clear, therefore, that if this time effect in filling were eliminated a satisfactory assay would be obtained. The simplest method of elimination would have been to make a constant correction to the potency for the first unknown, a similar correction of twice the magnitude to the second unknown, and a similar correction of three times the magnitude to the third unknown. Unfortunately, examination of a few plates showed that the magnitude of such corrections would not be constant for all plates. Another method of elimination would have been to substitute the standard for the third unknown, the standard being on the plate twice, once at the beginning and once at the end. This would allow a determination of the magnitude of the correction for each plate, but would reduce the number of unknowns being assayed from three per plate to two per plate. The cost per assay would thus be increased by $50 \%$.

The use of the quasi-Latin square. Yates (1937) has given a design for carrying out an experiment of the form $2^{5}$ (five factors all at two levels) in an $8 \times 8$ square, which involves the partial confounding of the higher order interactions. This design can be adapted to our purpose. We consider that we have one factor at two levels (the high and low dilutions) and one factor at eight levels. This factor at eight levels can be regarded as made up as

(1) standard preceding unknowns,

(2) unknown 1 succeeding the standard by one time interval,

(3) unknown 2 succeeding the standard by two time intervals,

(4) unknown 3 succeeding the standard by three time intervals,

(5) unknown 3 preceding the standard by three time intervals,

(6) unknown 2 preceding the standard by two time intervals,

(7) unknown 1 preceding the standard by one time interval,

(8) standard succeeding the unknowns. 
This leads to an arrangement such as that in Table 7. Ideally this should be re-randomized every time it is used, but when doing 20-30 plates a day this is impracticable. The most satisfactory compromise is probably to construct about 20-30 arrangements, and have separate forms printed similar to Table 7 for each. Appropriate templates for transferring the results from the main table to the smaller one and for filling the plates would also be needed. The arrangement to be used in any particular assay can be selected with a table of random numbers. In Table 7, which has been used in all the assays of this type reported in this paper, the restriction has been imposed that in all rows and all columns the high and low levels alternate. This was to assist the operator in filling the plate, but a completely random arrangement is to be preferred on theoretical grounds. Randomization in practice can be carried out by drawing numbers from 1 to 8 from a hat, and rearranging the rows in the order thus given, and repeating this process for columns.

Table 7. Form of entry of results from quasi-Latin square, and an example of its use

Streptomycin assays

Assays: (1) $\left(S_{3}\right)$

(2) $\left(S_{1}\right)$

(3) (C)
Date............

Plate poured by

Plate filled by

Dilutions made by

Readings by.

Calculations by.

Checked by....

Dilutions of samples: (1) $1 / 2 \cdot 4$ and

(2) $1 / 36$

(1) $1 / 24$

(3) $1 / 40$

(2) $1 / 360$

(3) $1 / 400$

Concentrations of standard: 10 and 1 units $/ \mathrm{ml}$.

\begin{tabular}{|c|c|c|c|c|c|c|c|}
\hline & 4.4 & & & & & & \\
\hline & $1_{L}^{F} \quad 10 \cdot 5$ & & & & & & \\
\hline & $3_{H}^{l} \quad 14.2$ & $\cdot 2$ & & & & & \\
\hline & & & & & & & \\
\hline & & & 6 & 6 & & 4 & \\
\hline & & & $0 \cdot 1$ & & & & \\
\hline & & $10 \cdot 2$ & $13 \cdot 6$ & $10 \cdot 2$ & 13.9 & $0 \cdot 6$ & \\
\hline & & & & & & & \\
\hline
\end{tabular}

$\begin{array}{cccccccc}S_{L} & S_{H} & 1_{L} & 1_{H} & 2_{L} & 2_{H} & 3_{L} & 3_{H} \\ 11 \cdot 1 & 14 \cdot 9 & 10 \cdot 9 & 15 \cdot 2 & 11 \cdot 1 & 14 \cdot 4 & 10 \cdot 9 & 14 \cdot 6 \\ 11 \cdot 2 & 14 \cdot 5 & 10 \cdot 5 & 14 \cdot 6 & 11 \cdot 1 & 14 \cdot 2 & 10 \cdot 7 & 14 \cdot 0 \\ 10 \cdot 5 & 15 \cdot 1 & 10 \cdot 2 & 13 \cdot 7 & 10 \cdot 6 & 14 \cdot 5 & 10 \cdot 7 & 14 \cdot 2 \\ 10 \cdot 6 & 13 \cdot 7 & 10 \cdot 9 & 13 \cdot 5 & 10 \cdot 3 & 14 \cdot 5 & 10 \cdot 5 & 13 \cdot 9 \\ 10 \cdot 6 & 14 \cdot 0 & 10 \cdot 4 & 13 \cdot 5 & 10 \cdot 7 & 14 \cdot 4 & 10 \cdot 0 & 13 \cdot 6 \\ 10 \cdot 0 & 14 \cdot 2 & 10 \cdot 1 & 13 \cdot 5 & 10 \cdot 1 & 13 \cdot 5 & 10 \cdot 7 & 13 \cdot 2 \\ 10 \cdot 6 & 13 \cdot 6 & 10 \cdot 2 & 13 \cdot 9 & 10 \cdot 2 & 13 \cdot 6 & 10 \cdot 0 & 14 \cdot 1 \\ 10 \cdot 8 & 13 \cdot 6 & 10 \cdot 2 & 13 \cdot 5 & 10 \cdot 5 & 13 \cdot 7 & 10 \cdot 0 & 14 \cdot 0 \\ 85 \cdot 4 & 113 \cdot 6 & 83 \cdot 4 & 111 \cdot 4 & 84 \cdot 6 & 112 \cdot 8 & 83 \cdot 5 & 111 \cdot 6\end{array}$

Titre of samples: (1) $20 \cdot 3$ units/ml.

$$
\begin{aligned}
\mathbf{1}_{B}+\mathbf{1}_{L}-S_{H}-S_{L} & =D=-\mathbf{4} \cdot 2 \\
\mathbf{1}_{H}-\mathbf{1}_{L}+S_{H}-S_{L} & =B=\mathbf{5 6 \cdot 2} \\
\text { Antilog } D / B & =\mathbf{0} \cdot \mathbf{8 4 2 0} \\
& \\
\mathbf{2}_{H}+2_{L}-S_{H}-S_{L} & =D=-\mathbf{1} \cdot 6 \\
\mathbf{2}_{H}-2_{L}+S_{H}-S_{L} & =B=\mathbf{5 6} \cdot 4 \\
\text { Antilog } D / B & =\mathbf{0} \cdot \mathbf{9 3 6 8} \\
& \\
3_{H}+\mathbf{3}_{L}-S_{H}-S_{L} & =D=-\mathbf{3} \cdot 9 \\
\mathbf{3}_{H}-\mathbf{3}_{L}+S_{H}-S_{L} & =B=56 \cdot 3 \\
\text { Antilog } D / B & =\mathbf{0} \cdot \mathbf{8 5 2 6}
\end{aligned}
$$
(2) 337
(3) 341

,




\section{Preferred method of assay of streptomycin}

The following is the preferred method for dealing with about 60-90 assays (20-30 plates) per day. Sufficient nutrient agar (Bactopeptone, 5 g.; Difco beef extract, 3 g.; agar, 15 g.; distilled water to 1 l.; pH 8 before autoclaving at $15 \mathrm{lb}$. for $20 \mathrm{~min}$.) for all the plates $(140 \mathrm{ml} . /$ plate) is placed in a flatbottomed Pyrex flask fitted with a ground-glass interchangeable neck, and the whole sterilized in the usual way. The flask contents are cooled in a water-bath at $60^{\circ}$ for about 30-60 min. and then inoculated with a culture of $B$. subtilis. It has been found best to arrange that the inoculum is of approximately the same amount of the same age from day to day, and to this end agar slopes are prepared in $8 \mathrm{oz}$. culture bottles from $20 \mathrm{ml}$. of yeast-beef agar (above composition) which are sterilized at $15 \mathrm{lb}$. for $20 \mathrm{~min}$. before sloping. Three such bottles are inoculated daily from a subculture carried in a Freudenreich bottle, which in turn has been prepared from the master culture of $B$. subtilis 288 . Incubation of the slopes is at $32^{\circ}$ for 5 days. The daily inoculum for the agar for the plates is obtained by adding $20 \mathrm{ml}$. of sterile distilled water to each of two or three of the $8 \mathrm{oz}$. slopes and shaking to suspend the spores. The mixed suspension from two bottles is usually sufficient to inoculate 3-4 1. of agar.

The inoculated agar is maintained at $60^{\circ}$ for about $10-20 \mathrm{~min}$. to afford some degree of pasteurization. About $140 \mathrm{ml}$. of the agar is then run on to the plate. The latter is previously warmed to $60^{\circ}$ in an oven and removed as required for filling. The agar is poured mainly on to one part of the plate and run over the surface with as little tilting as possible; air bubbles are burst with a hot sterile needle. The plate covered with a glass sheet is laid on a perfectly flat table which is tested with a spirit level daily. The agar is usually completely set in $\mathbf{3 0} \mathrm{min}$. when disks are cut with a sterile no. $\mathbf{5}$ cork borer in positions determined by the plan marked on paper laid beneath the plate. The disks are removed with spear-shaped needles and placed in lysol.

The standard solution required for a week's work is made up to $100 \mathrm{units} / \mathrm{ml}$. in $\mathrm{pH} \mathrm{6.0} \mathrm{phosphate} \mathrm{buffer,} \mathrm{and} \mathrm{each} \mathrm{day} \mathrm{suitable} \mathrm{amounts} \mathrm{are} \mathrm{diluted} \mathrm{in}$ buffer $\mathrm{pH} \mathrm{8.0}$ to give solutions containing 10 and 1 units $/ \mathrm{ml}$. Unknown solutions are similarly diluted in $\mathrm{pH} 8.0$ buffer to give concentrations approximately 10 and 1 units/ml. (the ratio of concentrations of the two solutions must be $10: 1$ ).

The slopes of the $\log c$-diameter curves for the standard and unknowns are sometimes appreciably different, and it has been confirmed that the $\mathrm{pH}$ 's of such solutions often differ. The dilution of streptomycin sulphate solutions in $\mathrm{pH} 6.0$ buffer with buffer of $\mathrm{pH} 8.0$ gives solutions of $\mathrm{pH}$ about $7 \cdot 6$ or over. Many test solutions have $\mathrm{pH}$ values well above 8 , and when diluted in $\mathrm{pH} \mathrm{8.0}$ buffer the final value is about $8 \cdot 2-8 \cdot 4$. For very precise work it would undoubtedly be desirable to adjust the $\mathrm{pH}$ of the standard and unknown solutions exactly to $\mathbf{8 \cdot 0}$, but for routine research or production this would be too time-consuming.

Each operator uses her own cavity filler throughout. This consists of a 1-2 mm. capillary drawn out at each end and held by rubber tubing in a wider 
piece of glass tube, to the other end of which a rubber bulb is attached. The volume of the capillary should be about $0.05-0.06 \mathrm{ml}$. and experience will show the best shape for the jets. With a little care the capillary can be quickly filled and kept free from an air bubble, and by gently squeezing the bulb the contents of the capillary can be discharged into a cavity on the plate. Great importance is attached to ensuring that the same volume of liquid is placed in each cavity.

The operator has before her eight samples ('High' and 'Low' concentrations of each of three unknowns and the standard), and the plate lies on a special template based on Table 7 so arranged as to facilitate filling. The template is in fact Table 7 altered in scale to fit the actual plate. The rows (and columns) should be about $3.43 \mathrm{~cm}$. apart, giving a total width and height of $24 \mathrm{~cm}$. The terms $\mathbf{1}_{L}^{F}$, etc., are replaced by more vivid symbols. Thus four different colours can be used to represent the four different solutions, the early cavities and the late cavities can be represented by circles and triangles respectively, which can be made large and small to represent the high and low concentrations.

The filling order is as follows:

(1) The four cavities marked $S_{H}^{I}$, followed by the four cavities $S_{L}^{I}$. The suffixes $H$ and $L$ refer to the high and low concentrations and the superscript $I$ means that these sets of four are the initial entries for the standard. These correspond to level (1) in the list of the eight levels given in the previous section.

(2) The four cavities marked $1_{H}^{I}$, then the four cavities marked $1_{L}^{I}$, corresponding to level (2).

(3) and (4) similarly for unknowns (2) and (3).

(5) The four cavities $3_{L}^{F}$ and then the four cavities $3_{H}^{F}$, corresponding to level (5). The superscript $F$ means that these sets are the final entries for this sample.

(6), (7) and (8) similarly for unknowns (2) and (1) and the standard, corresponding to levels (6), (7) and (8).

Between each solution the filler is washed with $\mathrm{pH} 8$ buffer.

Filling is at first confusing, but with a little practice the operator soon acquires a steady rhythm, and after a few days filling becomes quite mechanical. It is essential that filling should proceed at a steady rate; a break of 2 or 3 min. makes the assay useless, whether in large-plate or Petri-dish work.

The plates are recovered and carefully placed in a horizontal position in a $32^{\circ}$ incubator. Next morning the zone diameters are measured. After checking doubtful readings, the agar is peeled off the plate into a bucket of lysol and the plates and frames are immersed for an hour in this disinfectant. After washing thoroughly they are wiped with acid ethanol and dried in the oven 1-2 hr. at $60^{\circ}$. No attempt is made to ensure perfect sterility.

For transferring the zone diameter readings from the main table to the lower one in Table 7 eight templates, corresponding to the arrangement in question, are useful for laying on the main table to isolate the eight readings of $S_{L}$, etc.

Calculation of the results is greatly assisted by an adding machine. The necessary subsequent steps are carried out according to the schemes given at 
the lower right-hand side of the typical record sheet in which the results of an actual assay have been inserted (Table 7). The three sections in this record refer to the three unknowns. $\mathbf{1}_{H}, \mathbf{1}_{L}, \mathrm{~S}_{H}$ and $\mathrm{S}_{L}$, etc., refer to the totals of the corresponding columns to the left of the record and antilog $D / B$ refers to the antilog to base 10 of the result obtained by dividing $D$ by $B$. (Sometimes $D / B$ is negative; for example, it might be -0.07473 . In this case one remembers that -0.07473 equals $\overline{\mathbf{1}} \cdot 92527$ and finds the antilog of this, $0 \cdot 8420$.) The antilog $D / B$ is the factor by which the concentration of the standards must be multiplied in order to give the concentrations of the unknowns (as placed in the cavities). Thus if the factor came out as $\mathbf{0} \cdot \mathbf{8 4 2 0}$, the high and low concentrations of the unknown placed on the plate are $10 \cdot 46$ and 1.046 units $/ \mathrm{ml}$. Of course, these must be multiplied by the dilution factor in order to give the concentration of the original solution; i.e. the unknown solution may have been diluted with $\mathrm{pH} 8 \cdot 0$ buffer to dilutions of $1 / 2 \cdot 4$ and $1 / 24$ in order to give concentrations approximately 10 and 1 units $/ \mathrm{ml}$. respectively. In this case the concentration of the original would be $24 \times \mathbf{0 . 8 4 2 0}=\mathbf{2 0} \cdot \mathbf{2} \mathrm{units} / \mathrm{ml}$. If the method is used for penicillin assays, it is usual to work with the agar at $\mathrm{pH} 7 \cdot 0$, to use solutions at $\mathrm{pH} 7 \cdot 0$, to use concentrations of standard of 0.5 and $2 \cdot 0$ units $/ \mathrm{ml}$. and similarly for the unknowns. In this case, the ratio of the high and low concentrations being $4: 1$, it is necessary to find antilog $0.6021 D / B$ instead of antilog $D / B$ to get the factor. In general, the factor is found from antilog $[(D / B) \times \log$ (ratio of concentrations) $]$ which for the streptomycin assays becomes antilog $D / B$.

A chart or set of tables can be constructed to give rapidly the value of the factor when $D$ and $B$ are known. Although at first confusing, the calculation, like filling the plate, soon becomes mechanical.

To illustrate the precision possible, Table 8 records the results obtained when three working standards $S_{1}, S_{2}, S_{3}$, and six solutions A to $\mathrm{F}$ were referred to a sample of pure streptomycin sulphate.

Table 8. Reproducibility of assay potencies obtained by the preferred method

$\begin{array}{rcccccccccc}\text { Plate } & \text { Standard } & S_{1} & S_{2} & S_{3} & A & B & C & D & E & F \\ 1 & 827 & 334 & 125 & - & 223 \cdot 5 & - & - & - & - & - \\ 2 & 827 & 334 & 118 & - & - & 673 & - & - & - & - \\ 3 & 827 & 341 \cdot 5 & 122 & - & - & - & 345 & - & - & - \\ 4 & 827 & 342 & 123 & - & - & - & - & 512 & - & - \\ 6 & 827 & 355 & 122 & - & - & - & - & - & - & 828 \\ 7 & 827 & - & 121 & 20 \cdot 7 & 216 & - & - & - & - & - \\ 8 & 827 & - & 124 & 20 \cdot 4 & - & 670 & - & - & - & - \\ 9 & 827 & - & 122 & - & - & - & 331 \cdot 5 & - & - & - \\ 10 & 827 & - & 121 & 20 \cdot 7 & - & - & - & 491 & - & - \\ 11 & 827 & - & 121 & 20 \cdot 6 & - & - & - & - & 81 & - \\ 12 & 827 & - & 120 & - & - & - & - & - & - & - \\ 13 & 827 & 340 & - & 20 \cdot 8 & 218 & - & - & - & - & - \\ 14 & 827 & 350 \cdot 5 & - & - & - & 680 \cdot 5 & - & - & - & - \\ 15 & 827 & 337 & - & 20 \cdot 2 & - & - & 341 & - & - & - \\ 16 & 827 & 334 & - & 20 \cdot 7 & - & - & - & 511 & 78 & - \\ 17 & 827 & 333 & - & 20 \cdot 0 & - & - & - & - & - & 898 \\ 18 & 827 & 344 \cdot 5 & - & 19 \cdot 6 & - & - & - & - & 79 \cdot 5 & 857 \\ & \text { Means } & 340 \cdot 5 & 122 & 20 \cdot 4 & 219 & 674 & 339 & 505 & 7 & \end{array}$


Analyses of variance of three of the plates chosen at random are given in Table 9. The parallelism term is the interaction between treatments and levels, and has a total of seven degrees of freedom. Of these, however, one is partially confounded with columns, leaving six. In all cases the row and column mean squares are significant, frequently highly so, showing that the Latin square arrangement is removing from the error term a large part of the effect of the inhomogeneity of the plate. The average residual is 0.01749 . It is interesting to note that the average residual obtained by ignoring the double restriction achieved by the Latin square, i.e. by pooling the sums of squares for rows, columns, and the residual, is $0 \cdot 13973$. The increase in accuracy through the use of the Latin square arrangement is given by the ratio of these residuals, viz. about $8 \cdot 0$ times.

Table 9. Analyses of variance of three plates selected from Table 8

\begin{tabular}{|c|c|c|c|c|c|c|c|}
\hline \multirow{2}{*}{$\begin{array}{c}\text { Source of } \\
\text { variance }\end{array}$} & \multirow{2}{*}{$\begin{array}{l}\text { Degrees } \\
\text { of } \\
\text { freedom }\end{array}$} & \multicolumn{2}{|c|}{ Assay no. 8} & \multicolumn{2}{|c|}{ Assay no. 15} & \multicolumn{2}{|c|}{ Assay no. 17} \\
\hline & & s.s. & M.S. & s.s. & M.s. & s.s. & M.s. \\
\hline Rows & 7 & 0.90610 & 944 & $6 \cdot 18859$ & 0.88408 & $3 \cdot 96000$ & 0.56571 \\
\hline Columns & 7 & 0.06110 & 0.00873 & 3.96859 & 0.56694 & $3 \cdot 62000$ & 0.51714 \\
\hline Treatments & 7 & - 1.19110 & $0 \cdot 17016$ & 0.81859 & 0.11694 & $1 \cdot 84750$ & 0.76393 \\
\hline Levels & 1 & $215 \cdot 72266$ & $215 \cdot 72266$ & $197 \cdot 75390$ & $197 \cdot 75390$ & $244 \cdot 14063$ & $244 \cdot 14063$ \\
\hline Parallelism & 6 & $0 \cdot 13219$ & 0.02203 & 0.37344 & 0.06224 & $0 \cdot 15125$ & 0.02521 \\
\hline Residual & 35 & 0.26170 & 0.00748 & 0.83423 & 0.02385 & $0 \cdot 74062$ & 0.02116 \\
\hline Tota & 63 & $218 \cdot 27485$ & & $209 \cdot 93734$ & & $254 \cdot 46000$ & \\
\hline
\end{tabular}

S.s. = sums of squares; M.s. = mean squares.

To calculate the internal error of an assay, we use the formula (cf. Finney, 1944)

$$
s_{m}=\frac{2 s d \sqrt{ } n}{B^{2}} \sqrt{ }\left(B^{2}+D^{2}\right),
$$

where $s_{m}$ is the standard error of the logarithm of the potency ratio, $s$ is the standard error of an individual observation, $d$ is the logarithm of the dilution ratio, $n$ is the number of observations at each of the four points of the assay, and $B$ and $D$ have the meaning given in Table 7 . In the case where the unknown has a potency close to that of the standard, $D$ is small compared with $B$, and we get

$$
s_{m}=\frac{2 s d \sqrt{ } n}{B}
$$

In the present case, an average value for $s$ is $\sqrt{ } \mathbf{0} \cdot 01749, d$ is $\log 10$ and therefore unity, $n$ is 8 , and $B$ is about 60 . The resulting value of $s_{m}$ is 0.0124 . For a large number of degrees of freedom we can take the $95 \%$ fiducial limits as $\pm 2 s_{m}$, or $\pm \mathbf{0 . 0 2 4 8}$. These are the limits on the logarithm of the potency ratio, so the limits on the potency ratio are 94.5 and 105.9 .

We can also obtain an estimate of the overall error from Table 8. For the $S_{1}, S_{2}, S_{3}$ observation the $95 \%$ confidence limits for a single assay are 
estimated as 96 and $104 \%$. The close agreement between the internal and external estimates makes it evident that there are no unsuspected sources of error.

Acknowledgements are due to the Distillers Company Ltd. for permission to publish this paper.

\section{REFERENCES}

Finney, D. J. (1944). Mathematics of biological assay. Nature, Lond., 153, 284.

Fisher, R. A. (1942). The Design of Experiments, 4th ed., Chapter v. Edinburgh: Oliver and Boyd.

Yates, F. (1937). The Design and Analysis of Factorial Experiments, p. 35. Harpenden Imperial Bureau of Soil Science.

(Received 9 July 1947) 